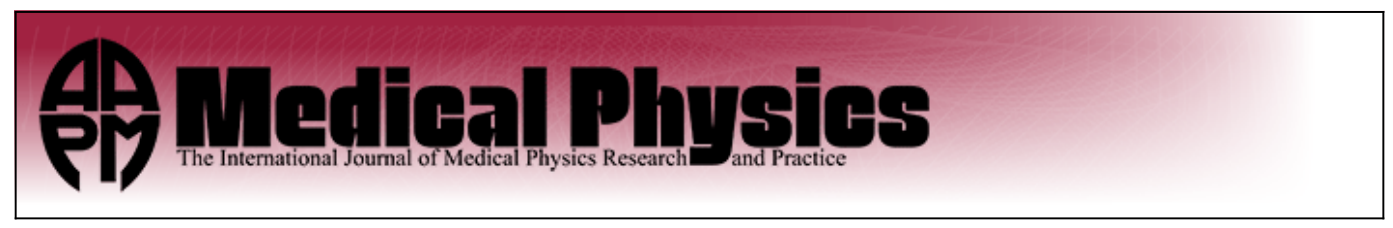

Structure and adaptation of arteries to pulsatile flow: The case of the ascending aorta Carla Silva and A. Heitor Reis

Citation: Medical Physics 41, 063701 (2014); doi: 10.1118/1.4876379

View online: $\mathrm{http}: / / \mathrm{dx}$.doi.org/10.1118/1.4876379

View Table of Contents: http://scitation.aip.org/content/aapm/journal/medphys/41/6?ver=pdfcov

Published by the American Association of Physicists in Medicine 


\title{
Structure and adaptation of arteries to pulsatile flow: The case of the ascending aorta
}

\author{
Carla Silva ${ }^{\text {a) }}$ \\ Polytechnic Institute of Tomar, Qta. do Contador, 2300-313 Tomar, Portugal and Évora Geophysics Centre, \\ R. Romão Ramalho, 59, 7000-671 Évora, Portugal \\ A. Heitor Reis ${ }^{\text {b) }}$ \\ Évora Geophysics Centre, R. Romão Ramalho, 59, 7000-671 Évora, Portugal and Physics Department, \\ University of Évora, R. RomãoRamalho, 59, 7000-671 Évora, Portugal
}

(Received 26 February 2014; revised 23 April 2014; accepted for publication 29 April 2014; published 21 May 2014)

Purpose: The objectives are: (i) assess the development of the impedance of some arteries during the first decades of life; (ii) determine the influence of pulse rate in arterial impedance; (iii) compare the structure of some arterial segments with optimized structures with respect to blood flow; and (iv) explain the elongation of the ascending aorta throughout life in healthy subjects.

Methods: A model of the arterial network previously developed by the authors, together with data of lengths, diameters, and distensibilities of arterial segments reported in the literature were used. The impedances of the aorta and carotid artery were calculated based on that model. Similarly, the impedances of various arteries corresponding to heart rates of $65 \mathrm{bpm}$ and $120 \mathrm{bpm}$ were calculated. Values observed in arterial segments were compared with the respective optimal values from the viewpoint of hemodynamic performance. This allowed drawing conclusions on the arterial segments that might be critical with regard to hemodynamics.

Results: It was found that in healthy people impedances of the aorta and the carotid artery decrease markedly with age especially during body growth. It was also found that impedances of the main arteries do not significantly change with heart rate, even if sharp changes in arterial distensibility are observed. With respect to optimal flow performance, it was found that scaling between diameters of branching arteries is generally close to optimality, while the corresponding length scaling is far from optimality. It was also found that the ascending aorta and aortic arch are among those arterial segments whose lengths are much smaller than the optimum values. An explanation is offered for the age associated elongation of the aorta in healthy people.

Conclusions: In healthy subjects, the human arterial system continues to optimize its performance at least until the age of 60. () 2014 American Association of Physicists in Medicine. [http://dx.doi.org/10.1118/1.4876379]

Key words: adaptation arteries pulsatile flow, elongation ascending aorta

\section{INTRODUCTION}

As part of the circulatory system, the heart rhythmically pumps blood throughout the arterial tree. In the arterial tree of chordate animals, the flow of blood is pulsatile in the sense that flow rate varies periodically around a mean value.

We may question whether pulsatile flow presents advantages over continuous flow in the transport of blood to tissues? Indeed, we can point to at least two reasons: (1) Studies have shown the benefits of pulsatile flow in kidney function, lymph flow, and oxygen consumption, which are increased during pulsatile perfusion; $;^{1,2}$ (2) pulsatile flow with distention of the arterial wall allows reducing peak pressure and also the energy expended by the heart to pump blood. Because the arterial wall distends during the propagation of the peak of the pressure wave, thereby increasing the cross section of the vessel, not only the flow resistance is reduced as also it allows storage of elastic energy, which makes it possible to maintain a negative gradient along the vessel needed to keep the flow subsequent to systole. This is especially true for the arteries that accommodate blood surging from the left ventricles during systole (aorta, brachiocephalic, right and left common carotid arteries, right and left subclavian arteries, right and left vertebral arteries, right and left common iliac arteries) (see Ref. 3).

Besides, blood also presents unique rheological properties. In fact, blood viscosity decreases with shear rate. This attribute together with dilation of the arterial wall which is increased at the sites of high shear rate (through production of nitric oxide) facilitates blood flow when it is at peak. These features are part of a much complex control system that operates on the cardiovascular system through the conjugate action of the cardio-acceleratory, cardio-inhibitory, and vasomotor centers, and that provides blood to individual organs and tissues according to immediate needs.

The human circulatory system operates under a large set of constraints, ranging from the shape of the body, the location of its organs and tissues to the temporary needs related to physiology or human activity. Many works have been 
devoted to modeling of the circulatory system (see for instance Refs. 4-10, and namely in recent years. ${ }^{3,11-13}$

Here we analyze what would be the optimal structure of certain parts of the human circulatory system as if it had the freedom to change their morphology to optimize its performance. To carry out this exercise is important because freedom to morph exists at some degree during growth in childhood and adolescence, and also during the entire lifetime in some vessels that are not specially constrained by the nearby organs and tissues as it is the case of the ascending aorta. As we show in this paper, it seems likely the shape of the ascending aorta is adjusted during lifetime in order to facilitate blood flow.

We also analyze the importance of pulsatile flow frequency on blood flow impedance. With this purpose we use the resistive-capacitive (RC) model developed by Silva and Reis ${ }^{14}$ which provides relationships that involve pulse frequency (heart rate), vessel diameter and length, arterial distensibility, and blood viscosity.

\section{BLOOD FLOW IMPEDANCE THROUGOUT LIFETIME}

In the case of pulsatile flow in a vessel, the ratio of inlet/outlet pressure difference to flow rate measures its impedance, i.e., the extent to which the vessel resists the passage of a pulsed flow of blood. In case of a vessel of diameter $D$ and length $L$, (aspect ratio $x=L / D$ ) with pulsatile flow of frequency $\omega$, impedance reads (see Ref. 14):

$$
|Z|=k_{A} x y^{-1}\left(1+\omega^{2} / \hat{\omega}^{2}\right)^{-1 / 2},
$$

where $k_{A}=128 \mu \pi^{-1}, \mu$ is dynamic viscosity of the fluid, $y=D^{3}, k=k_{A} \pi \beta / 4, \beta=(2 / D)(d D / d P)$ is the distensiblity coefficient of the vessel wall, $P$ is pressure, and $\hat{\omega}=1 / k x^{2}$ represents the characteristic frequency of the vessel. From Eq. (1), it is evident that impedance decreases with frequency (heart rate) and vessel diameter, and increases with vessel length and characteristic frequency. Because characteristic frequency varies inversely with $\beta$, impedance decreases with the distensiblity coefficient of the vessel wall, therefore showing the importance of this parameter to ease blood flow.

Impedance as calculated from Eq. (1) results from a RC model of pulsatile flow $^{14}$ based on the Navier-Stokes equation for unidirectional flow: $\partial u / \partial t+u \cdot \operatorname{grad} u=-\rho^{-1} \operatorname{grad} P$ $+v$ lap $u$, in which the inertial terms have been discarded because they are, at least, of one order of magnitude smaller than the other terms, as it is shown through scale analysis. In this way, let $u$ denote average blood velocity, $\tau$ characteristic time related to pulse wave frequency, $L_{c}$ the characteristic length in the flow direction, $D$ vessel diameter, $\rho$ blood density, $\Delta P$ pressure drop along the vessel, and $v$ blood kinematic viscosity. Then, by assuming the following scale values for large arteries: $u \sim 10^{-1} \mathrm{~m} \mathrm{~s}^{-1}, \tau \sim 1 \mathrm{~s}, L_{c} \sim 1 \mathrm{~m}, D$ $\sim 10^{-3} \mathrm{~m}, \Delta P \sim 10^{3} \mathrm{~Pa}$, and $v \sim 10^{-5} \mathrm{~m}^{2} \mathrm{~s}^{-1}$, the orders of magnitude (in $\mathrm{ms}^{-2}$ ) of the terms in the Navier-Stokes equation are: $\partial u / \partial t \sim 10^{-1}, u$.grad $\mathrm{u} \sim 10^{-2}, \rho^{-1} \operatorname{grad} P \sim 1$, $\rho^{-1}$ lap $u \sim 1$, therefore justifying the use of the RC model ${ }^{14}$ as a first approach in the study of the human arterial system. Models that include the term $\partial u / \partial t$ lead to greater complexity in the calculations but did not cause a change in the conclusions. For example, the RLC model developed by Jager et $^{\mathrm{al}}{ }^{5}$ accounts for the "sleeve effect," which arises from the interaction between viscous and inertial terms in the Navier-Stokes equation. However, in the same study, ${ }^{5}$ it was shown that the "sleeve effect" is important in some arteries at frequencies higher than $15 \mathrm{rad} \mathrm{s}^{-1}$, which is somehow beyond the normal range of the human pulse frequency.

Therefore, Eq. (1) enables calculation of blood flow impedances of some arteries for which data are available, namely, the aorta and the carotid artery. With this purpose we used the normal values of cross-sectional area and distensibility of the aortas of healthy children and young adults that serve as a reference for the detection of pathological changes of the aorta in case of disease. ${ }^{15}$ We also used data of normal length of the aorta of healthy adults (aged from 19 to 79 years), which exhibits a nearly linear increase with age ${ }^{16}$ therefore allowing the estimation of the values in the range $0-18$ years. With respect to the carotid artery we used data of normal diameters ${ }^{17}$ and distensibilities, ${ }^{18}$ and of normal lengths ${ }^{16}$ found in the literature. Blood viscosity changes with shear stress, therefore is not easy to assign it a definite value in pulsatile flow. However, we used the values in Ref. 19 to assign an average value to each vessel, according to the respective diameter. The heart rate considered was 65 beats per minute (bpm). The relative error affecting the calculation of impedances is given by: $|(\Delta|Z|) /| Z|| \approx|(4 \Delta D) / D|$ $+|(\Delta L) / L|+\left|[(\Delta \hat{\omega}) / \hat{\omega}](\omega / \hat{\omega})^{2}\right| \quad \operatorname{Because}(\omega / \hat{\omega})^{2} \sim 10^{-4}$, the contribution of the characteristic frequency to the error may be neglected. The relative error $(\Delta D) / D$ is of order $10^{-1}$ (see Refs. 15 and 17), while $(\Delta L) / L$ is of order $10^{-1}$ or smaller. $^{16}$ Therefore, the relative error affecting the impedances calculated from Eq. (1) may reach 50\%. However, this exercise is important because it allows verify if changes in impedance of the aorta of healthy humans occur during lifetime. The results for the ascending aorta, the descending aorta, and the carotid artery are shown in Figs. 1 and 2 , respectively.

It is shown that the descending aorta presents the highest relative impedance, followed by the carotid, and then by the

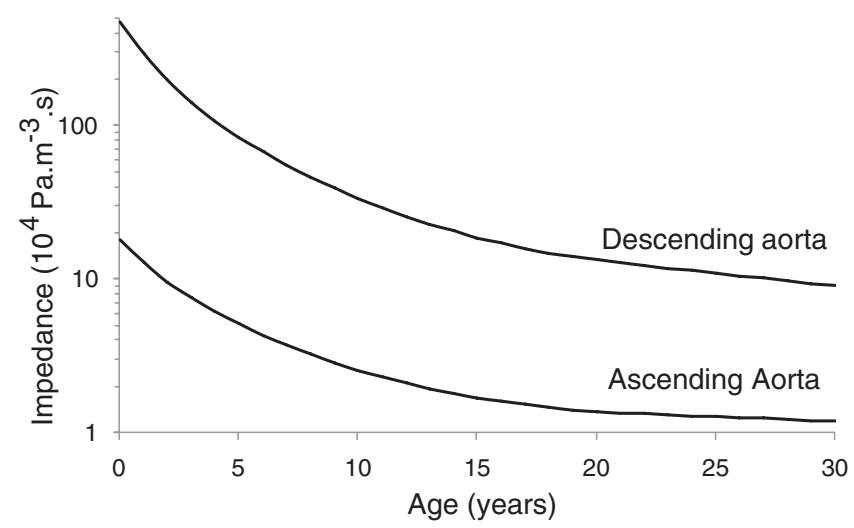

FIG. 1. Impedances of the ascending aorta and the descending aorta between birth and 30 years of age. 


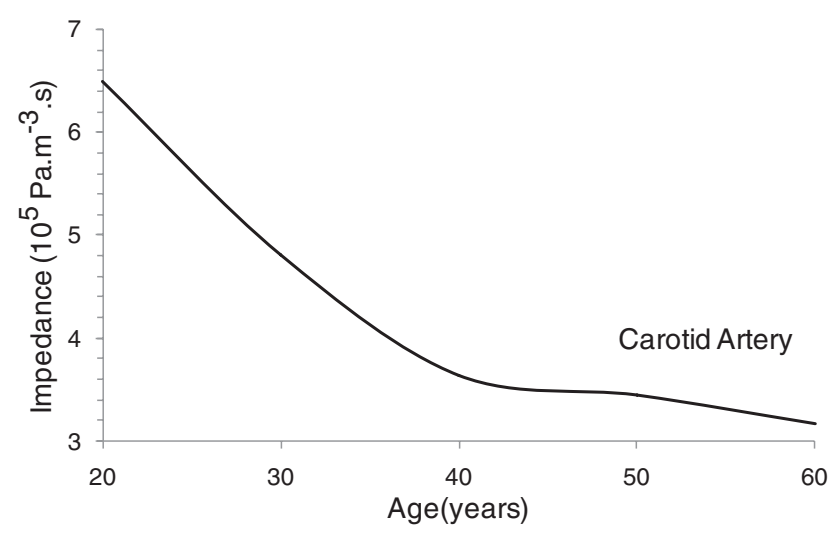

FIG. 2. Impedances of the carotid artery between 20 and 60 years of age.

ascending aorta. In fact, this hierarchy of impedances was expected because, in a flow tree, daughter vessels present impedances higher than that of parent vessel.

We also observe that in healthy humans the impedances of these arteries noticeably decrease with age, a trend that due to the magnitude of the values involved is not invalidated by the somewhat high relative error affecting the calculations. However, there are some differences: the impedances of both the ascending and the descending aorta show a continuous reduction that is very pronounced during growth of the human body ( $0-20$ years), and less marked between 20 and 30 years of age. Due to lack of data, we were not able to estimate aorta impedances subsequent to the third decade of life. After birth and until the fourth decade, the impedance of the carotid decreases linearly with age, and then it keeps decreasing until the age of 60 .

O'Rourke and Hashimoto ${ }^{11}$ found that at all heart rates the average ascending aortic impedance at the age of 80 is higher than at the age of 20. In fact, our study on impedance of the ascending aorta is focused on the period between birth and the age of 30. What our results show (Fig. 1) is that in healthy people the ascending aortic impedance decreases in that period. We believe that at the age of 80 arterial stiffening (together other aging processes) dominates over the processes that might be in place to improve arterial performance.

Then a question arises: why the impedances of the main vessels decrease with age? One can find a rationalization in the thought that Nature optimizes the human arterial tree in time, as it is observed in many natural systems. In fact, there is a large body of works that point to this direction. This tendency was summarized in a principle of maximization of "global flow access" known as the Constructal Law, which was first put forward in 1997 by Bejan $^{20}$ in the form: "For a finite-size system to persist in time (to live), it must evolve in such a way that it provides easier access to the imposed (global) currents that flow through it." The Constructal Law entails generation of flow configuration such that it provides the highest global conductivity compatible with the existing constraints, and has successfully explained shapes and patterns of many both animate ${ }^{21,22}$ and inanimate ${ }^{23-25}$ systems (see also some Constructal Law reviews $^{26-29}$ ).
TABLE I. Arterial impedances $\left(\mathrm{Pa} \mathrm{m}^{-} 3 \mathrm{~s}\right)$ of some peripheral arteries as function of heart rate.

\begin{tabular}{lcc}
\hline \hline & \multicolumn{2}{c}{ Heart rate } \\
\cline { 2 - 3 } Arteries & $65 \mathrm{bpm}$ & $120 \mathrm{bpm}$ \\
\hline Subclavian B & $2.3937 \times 10^{+07}$ & $2.3840 \times 10^{+07}$ \\
Radial & $4.5824 \times 10^{+08}$ & $4.5403 \times 10^{+08}$ \\
Femoral & $1.9486 \times 10^{+08}$ & $1.8873 \times 10^{+08}$ \\
Ulnar B & $7.2940 \times 10^{+08}$ & $7.2500 \times 10^{+08}$ \\
Posterior tibial & $1.5063 \times 10^{+09}$ & $1.3992 \times 10^{+09}$ \\
Anterior tibial & $3.3816 \times 10^{+09}$ & $2.8940 \times 10^{+09}$ \\
\hline \hline
\end{tabular}

\section{PULSE FREQUENCY AND BLOOD FLOW IMPEDANCE IN THE PERIPHERAL ARTERIES}

Another aspect that deserves attention is the importance of pulse frequency on impedance. We used Eq. (1) together with the data of diameter, length and distensibility of each vessel provided in Ref. 3 to study the influence of pulse frequency on the arterial impedance of 43 arterial segments. The study was conducted through comparing arterial impedances corresponding to 65 and $120 \mathrm{bpm}$.

By using vessel distensibilities presented in Ref. 3, for the most part of the arteries studied no noticeable differences were found in the respective impedances at each of these heart rates. This is due to the fact that the characteristic frequency $\hat{\omega}$ is much higher than $65 \mathrm{bpm}$, i.e., $\omega / \hat{\omega} \ll 1$. The few exceptions found, respect to some peripheral arteries (subclavian, radial, femoral, ulnar, posterior tibial, and anterior tibial) whose impedances decreased significantly as heart rate changed from 65 to $120 \mathrm{bpm}$ (see Table I).

As in the case of Figs. 1 and 2, the relative error affecting the impedances in Table I is of order 50\%, mainly due to the contributions of the relative errors respecting to arterial diameters and lengths. However, the trend observed when the heart rate changes from $65 \mathrm{bpm}$ to $120 \mathrm{bpm}$ is due mainly to the term $\left|[(\Delta \hat{\omega}) / \hat{\omega}](\omega / \hat{\omega})^{2}\right|$, for the reason that the characteristic frequency of the arteries in Table I is now close to the radial frequency $\omega$ corresponding to $120 \mathrm{bpm}$.

Especially, the decrease in impedance is higher in the major arteries of the legs (femoral, posterior tibial, anterior tibial). However, some studies show that arterial distensibility decrease with heart rate. ${ }^{30,31}$ Unfortunately, few data relating distensibility with heart rate are found in the literature. With regard to peripheral arteries data of the distensibilities of the radial and carotid arteries corresponding to 63, 90, and $110 \mathrm{bpm}$ were found in a seminal study by Giannattasio et al. ${ }^{30}$ If those data are used in Eq. (1), it is found that both the radial and carotid artery impedances do not change significantly from 63 to $110 \mathrm{bpm}$. An explanation based on performance optimization that involves the characteristics of the end capillaries of the tissues bathed by those arteries will be provided in a next paper by the authors.

\section{OPTIMAL DESIGN VERSUS ACTUAL SHAPE OF THE ARTERIES}

Murray's Law ${ }^{32-34}$ which states that the "cube of the radius of a parent vessel equals the sum of the cubes of the radii 
TABLE II. Observed average diameters and lengths of parent (in bold) and daughter vessels (within brackets), and values of daughter vessels (in italic) for optimal hemodynamic performance. The numbers identify vessels according to the scheme in Ref. 3. (p)—proximal diameter, (d)—distal diameter.

\begin{tabular}{|c|c|c|c|c|c|}
\hline Artery & Diameter (mm) & Length (mm) & Artery & Diameter (mm) & Length (mm) \\
\hline 3 brachiocephalic (d) & 18.0 & 34.0 & 50 external iliac (d) & 6.1 & 144 \\
\hline 4 subclavian A (p) & $6.4[11.5]$ & $12.1[34]$ & 52 femoral $(\mathrm{p})$ & $5.8[5.2]$ & $138.3[443]$ \\
\hline 5 common carotid (p) & $17.7[13.5]$ & $33.5[94]$ & 53 deep femoral (p) & $1.7[4.0]$ & $39.3[126]$ \\
\hline 4 subclavian A (d) & 9 & 34.0 & 52 femoral (d) & 3.8 & 443 \\
\hline 7 subclavian B (p) & $8.8[8.1]$ & $33.4[422]$ & 55 anterior tibial $(\mathrm{p})$ & $2.8[2.6]$ & $339.8[343]$ \\
\hline 6 vertebral $(p)$ & $3.1[3.7]$ & $11.8[149]$ & 54 posterior tibial $(\mathrm{p})$ & $2.7[3.1]$ & $318.0[321]$ \\
\hline 7 subclavian B (d) & 4.7 & 422 & 15 common carotid (d) & 6.0 & 139 \\
\hline 8 radial (p) & $5.0[3.7]$ & $441.8[235]$ & 16 internal carotid (p) & $6.0[5.3]$ & $138.4[178]$ \\
\hline 9 ulnar A (p) & $1.4[3.7]$ & $125.9[67]$ & 17 external carotid (p) & $1.4[4.7]$ & $31.9[41]$ \\
\hline 9 ulnar A(d) & 3.4 & 67 & 17 external carotid (d) & 4.3 & 41 \\
\hline 10 interosseous (p) & $1.5[2.1]$ & $30.0[79]$ & 85 external carotid (p) & $2.4[4.0]$ & $23.2[61]$ \\
\hline 11 ulnar B (p) & $3.3[3.2]$ & $64.9[171]$ & 86 sup. thy. asc. ph.lyng.(p) & $4.0[2.0]$ & $38.4[101]$ \\
\hline 14 aortic $\operatorname{arch} 2(\mathrm{~d})$ & 20.8 & 39 & 85 external carotid(d) & 3.5 & 61 \\
\hline 18 thoracic aorta $1(\mathrm{p})$ & $19.2[19.0]$ & $35.9[52]$ & 89 superficial temporal (p) & $2.2[3.2]$ & $37.5[61]$ \\
\hline 19 subclavian $\mathrm{A}(\mathrm{p})$ & $12.5[8.5]$ & $23.5[34]$ & 90 maxillary $(\mathrm{p})$ & $3.2[2.2]$ & $55.9[91]$ \\
\hline 41 abdominal aorta (d) & 10.4 & 20 & 89 superficial temporal (d) & 3 & 61 \\
\hline 42 common iliac (p) & $8.2[7.9]$ & $15.9[59]$ & 93 superftemp frontal $(p)$ & $2.4[2.2]$ & $48[100]$ \\
\hline 43 common iliac (left) (p) & $8.2[7.9]$ & $15.9[59]$ & 94 superftemp parietal $(p)$ & $2.4[2.2]$ & $48,5[101]$ \\
\hline 42 common iliac $(\mathrm{d})$ & 7.0 & 59 & 73 middle cerebral M1 (d) & 2.8 & 8 \\
\hline 50 external iliac (p) & $6.9[6.4]$ & $58.2[144]$ & 74 MCA M2 sup. (p) & $2.2[2.0]$ & $6.4[71]$ \\
\hline 51 inner iliac (p) & $2.4[4.0]$ & $20.2[50]$ & 75 MCA M" inf. (p) & $2.2[2.0]$ & $6.3[70]$ \\
\hline
\end{tabular}

of the daughters" stays as a landmark scaling law of geometries of branching channels with nonturbulent flows was originally proposed by Cecil D. Murray (1926) for the circulatory and respiratory systems, but was later proven to be valid for all laminar branching flows. ${ }^{3,4,21,27}$

In a previous work, ${ }^{14}$ based on minimization of global impedances, the authors derived scaling laws that generalize Murray's law for branching pulsatile flow. Hence, with respect to diameters the optimal scaling reads:

$$
\frac{D_{1}}{D_{0}}=\theta^{1 / 6}\left(1+\xi^{3}\right)^{-1 / 3}, \quad \frac{D_{2}}{D_{0}}=\theta^{1 / 6}\left(1+\xi^{-3}\right)^{-1 / 3},
$$

where $D_{0}$ is diameter of parent vessel, while $D_{1}$ and $D_{2}$ refer to diameters of daughter vessels, and

$$
\theta=\left(2 A_{i}^{-3 / 2}-A_{i}^{-1 / 2}\right) /\left(2 A_{0}^{-3 / 2}-A_{0}^{-1 / 2}\right), \quad i=1,2
$$

with $A_{i}=1+\omega^{2} k_{i}^{2} x_{i}^{4}, i=0,1,2$, and $\xi=L_{2} / L_{1}$ standing for branching asymmetry.

In a similar way, optimal scaling for artery lengths reads:

$$
\frac{L_{1}}{L_{o}}=\phi\left(1+\xi^{3}\right)^{-1 / 3}, \quad \frac{L_{2}}{L_{o}}=\phi\left(1+\xi^{-3}\right)^{-1 / 3}
$$

where $L_{0}$ is length of parent vessel, $L_{1}$ and $L_{2}$ refer to lengths of daughter vessels, and

$$
\phi=\theta^{2 / 3}\left(\frac{A_{1}}{A_{0}}\right)^{1 / 2} \text {. }
$$

As discussed in Sec. 3, except for some peripheral arteries (subclavian, radial, femoral, ulnar, posterior tibial, and anterior tibial), the characteristic frequency $\hat{\omega}$ is much higher than that corresponding to $65 \mathrm{bpm}$, i.e., $\omega / \hat{\omega} \ll 1$, and therefore optimal scaling is not much different from that of continuous flow. However, in Eqs. (2) and (4), the asymmetry parameter $\xi$ assigns smaller diameters and lengths, respectively, to the smaller daughter arteries. In effect, from Eqs. (2) and (4), one obtains:

$$
\frac{D_{2}}{D_{1}}=\frac{L_{2}}{L_{1}}=\xi,
$$

We tested the scaling laws [Eqs. (2) and (4)] by using the real values of various arterial bifurcations. For this purpose we used the extensive dataset of diameters, lengths and distensibilities of arteries provided in Ref. 3. We kept the identification number of the various arterial segments used in Fig. 2 and Table II of Ref. 3. The lengths in Eq. (4) respect to arterial segments that converge at a bifurcation. On the values given in Ref. 3, we have no absolute guarantee that some of these segments do not have small (or very small) intermediate branches. This can occur especially in very long segments in which it is likely to find small branches. A sign of this is that the proximal and distal diameters of these segments differ a lot. For this reason, the data for lengths of arterial segments are used with some caution. Because the relative error affecting data of both diameters and lengths is of order $10 \%$, while $\xi$ presents a relative error of same order, diameters and lengths of daughter vessels calculated through Eqs. (2) and (4) is of order $20 \%$. The results are shown in Table II.

In general, the diameters of daughter vessels calculated through Eqs. (2) are close to the measured ones. Yet, there are some exceptions (ulnar A, inner iliac, deep femoral, external carotid, and "sup. thy. asc. ph. lyng. fac. occ"). These daughter vessels have long parent vessels which may have small branchings, and therefore the respective data lengths might not be eligible in terms of the Eqs. (2). 
We also evaluated optimal length scaling and compared the calculated lengths of daughter vessels with the real ones. Here we can observe few agreements together with many discrepancies. As discussed before, there is some uncertainty about the data of lengths of the arterial segments used in the calculation, namely, if they correspond to segment free of small lateral branchings. This is especially true for the longer segments, as for instance the femoral artery $(443 \mathrm{~mm})$ in which the proximal diameter $(5.2 \mathrm{~mm})$ is much different from the distal diameter $(3.8 \mathrm{~mm})$ therefore indicating that many small branchings are likely to occur along the artery.

The scaling defined by the Eqs. (2) and (4) is optimized with respect to fluid (blood) dynamics only. However, many other constraints are in place, namely the distribution of organs in the body, together with their needs of blood supply, which were not considered in the derivation of those equations. Nevertheless, the exercise of comparing the actual values with the respective optimal values as regards blood flow is important since it identifies the arteries where blood flow is not optimized and where hemodynamics might affect arterial performance. Among such arterial segments are the ascending aorta, and the aortic arch that will be the object of Sec. 5. In Table II, we can identify other arterial segments that share such condition: common carotid, subclavian A, radial, ulnar A, inner iliac, deep femoral, external carotid, and "sup. thy. asc. ph. lyng. fac. occ." The segments that deviate from the optimal are likely to present increased hemodynamic resistances, namely, through increased shear stresses affecting vessel walls. Interestingly, the human body has control mechanisms that deal with this problem through increased production of nitric oxide in the vessel walls where shear stress is higher. ${ }^{35}$

\section{THE ELONGATION OF THE ASCENDING AORTA}

As referred before the ascending aorta is not optimized, as its length does not scale optimally [see Eq. (4)] with those of the aortic arch and the thoracic aorta A (see Fig. 3). In fact, while Eq. (4) calls for smaller lengths of daughter vessel segments with respect to parent ones, in the aorta occurs just the reverse. One may wonder why the chief vessel in the human body is not optimized with respect to blood flow. We believe that the special morphology of the aorta is the result of a trade-off among many objectives it has to comply with. In fact, in the small space allocated to it, the aorta has to divert the blood flow to the head, the arms, and organs in the thorax prior to redirecting the remaining flow to the rest of the body. This confers the aorta its special morphology, while it leaves it vulnerable to an extreme hemodynamic stress.

To investigate the optimal morphology of the aorta with respect to hemodynamics, we used Eq. (6). Then, by considering the diameter and length of the thoracic aorta, together with the diameter of the aortic arch B, we calculated the optimal length of the aortic arch B (see Fig. 3).

Then, in a similar way, by using the respective vessel diameter we successively calculated the lengths of the aortic $\operatorname{arch} \mathrm{A}$, and the ascending aorta. The values of the distensibil-

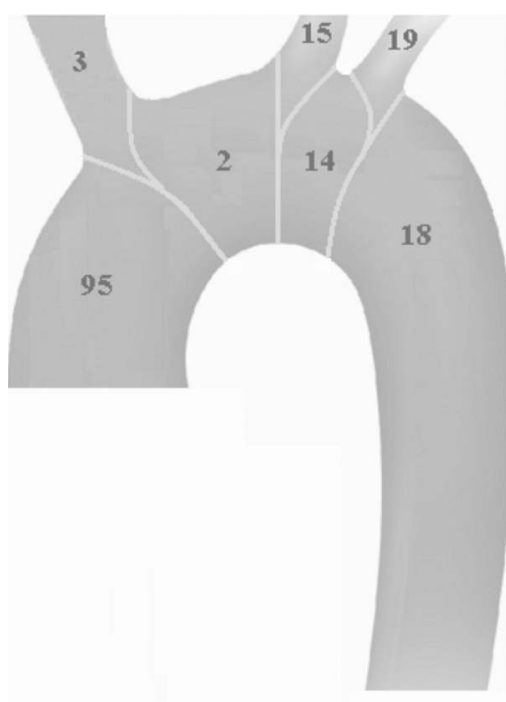

FIG. 3. Aorta segments and branches: 95-ascending aorta; 3brachiocephalic; 2-aortic arch A; 15-common carotid; 14-aortic arch B; 19-subclavian A; and 18-thoracic aorta A. The numbers identify vessels according to the scheme in Ref. 3 .

ities, diameters, and lengths of those vessels were taken from Ref. 3. The results are shown in Table III.

We observe that in order to perform optimally all these arterial segments would have to be longer. This is especially noticeable for the aortic arch A-which would have to be more than three times longer-and the ascending aorta that should have its length doubled.

Studies on the variation of the length of the ascending aorta have shown that the aorta lengthens with age, even in healthy humans, primarily due to the elongation of the ascending aorta. The lengths of the descending aorta and carotid and iliac arteries were not associated with age. ${ }^{16}$ In that study, it was shown that the average length of the ascending aorta (defined as from aortic annulus to apex of arch) increased from 44 to $98 \mathrm{~mm}$ between 20 and 80 years of age, according to the regression formula $L=0.90 Y+26.1 \mathrm{~mm}$ where Y stands for years of age.

In a comment to these results, O'Rourke et al. ${ }^{36}$ suggested that the elongation of the ascending aorta was due to pulsatile strain in the longitudinal direction of the proximal aorta that is greater than pulsatile strain in the circumferential direction, therefore one would expect greater lengthening with age in the longitudinal direction than increase in diameter.

Here we offer an alternative explanation based on the fact that both the ascending aorta and the aortic arch are not

TABLE III. Optimal average diameters and lengths of several segments of the aorta taking thoracic aorta $\mathrm{A}$ as the reference.

\begin{tabular}{lccc}
\hline \hline Artery & $\mathrm{D}(\mathrm{mm})$ & $\mathrm{L}(\mathrm{mm})$ & Lopt $(\mathrm{mm})$ \\
\hline 18 thoracic aorta A & 19.5 & 52 & $\ldots$ \\
14 aortic arch B & 21.1 & 39 & 56.2 \\
2 aortic arch A & 24.6 & 20 & 65.5 \\
95 ascending aorta & 29.4 & 40 & 78.3 \\
\hline \hline
\end{tabular}


optimized with respect to blood flow dynamics. Because, contrarily to other arterial segments that cannot morph in time for the reason that either they are associated to skeletal muscle or to bones of fixed length (as for instance, the arteries: radial, Ulnar A, inner iliac, deep femoral), the ascending aorta is quite free to morph because it is slightly constrained by adjacent tissues. As an approximate measure of the length from aortic annulus to apex of arch we add the optimal values of the aortic arch A and the ascending aorta to get $143.8 \mathrm{~mm}$ (see Table III), value that is the reference for optimal hemodynamics (minimum impedance). Therefore, in our view the elongation of the ascending aorta corresponds to optimization of the aortic morphology during lifetime towards an increasingly better hemodynamic performance. In this way, the aorta uses the freedom to morph for reducing the shear stresses associated to blood flow, and consequently the stresses that are induced in the arterial wall.

Morphing in time is a characteristic of the human body that seeks for better performance through adaptation either to external or internal constraints.

\section{CONCLUSIONS}

Based on data of arterial diameter and length, and distensibility coefficient after birth and at up to the age of 30 years it is shown that in healthy humans flow impedances of the ascending aorta and descending aorta decrease markedly in this period, while by using similar data for the period 0-60 years of age it is shown that impedance of the carotid artery keeps decreasing in this period.

It is also shown that flow impedances of arteries do not significantly change with heart rate. Despite distensibilities of some peripheral arteries-subclavian, radial, femoral, ulnar, posterior tibial, and anterior tibial—decrease as heart rate increases from 65 to $120 \mathrm{bpm}$, their respective impedances do not significantly change with heart rate.

By applying the scaling laws for minimum flow impedance, it is shown that artery diameters are in general quite close to the optimal values, while artery lengths are not. Among the arteries whose lengths are far from the optimal values are the ascending aorta, aortic arch, common carotid, subclavian A, radial, ulnar A, inner iliac, deep femoral, external carotid, and "sup. thy. asc. ph. lyng. fac. occ." This aspect was explained by the fact that arteries have to deliver blood to organs that are distributed in the body according to overall body performance, and therefore often do not comply with optimal performance with respect to blood flow. On the other hand, may be subject to additional stresses because they are not optimized hemodynamically.

Finally, it is shown that the normal lengths of the ascending aorta and the aortic arch are smaller than their optimal lengths. The optimal length of the ascending aorta plus the aortic arch is found to be $143.8 \mathrm{~mm}$. We interpreted the results of the study by Sugawara et al. ${ }^{16}$ that showed that in healthy individuals the average length of the ascending aorta (defined as from aortic annulus to apex of arch) increased from 44 to $98 \mathrm{~mm}$ between 20 and 80 years of age, as the tendency of this flow system to optimize its performance in time taking advantage of its freedom to morph due to be slightly constrained by adjacent tissues. This tendency has also been observed in many natural both animate and inanimate systems, and is known as the Constructal Law.

The results of this study may be extended to other animals provided that the relevant data are available.

\section{ACKNOWLEDGMENTS}

The authors acknowledge the funding provided by the CGE, under the contract Pest/OE/CTE/UI0078/2014, with FCT (the Portuguese Science and Technology Foundation).

a)Electronic mail: carlasilva@ipt.pt

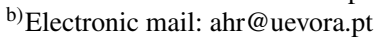

${ }^{1}$ C. Mavroudis, "To pulse or not to pulse," Ann. Thorac. Surg. 25, 259-271 (1978).

${ }^{2}$ K. M. Taylor, W. H. Bain, K. G. Davidson, and M. A. Turner, "Comparative clinical study of pulsatile and non-pulsatile perfusion in 350 consecutive patients," Thorax 37, 324-330 (1982).

${ }^{3}$ P. Reymond, F. Merenda, F. Perren, D. Rüfenacht, and N. Stergiopulos, "Validation of a one-dimensional model of the systemic arterial tree," Am. J. Physiol. 297, H208-H222 (2009).

${ }^{4}$ J. R. Womersley, "Method for the calculation of velocity, rate of flow and viscous drag in arteries when the pressure gradient is known," J. Physiol. 127, 553-563 (1955).

${ }^{5}$ G. N. Jager, N. Westerhof, and A. Noordergraaf, "Oscillatory flow impedance in electrical analog of arterial system: Representation of sleeve effect and non-Newtonian properties of blood," Circ. Res. 16, 121-133 (1965).

${ }^{6}$ W. R. Milnor, C. R. Conti, K. B. Lewis, and M. F. O'Rourke, "Pulmonary arterial pulse wave velocity and impedance in man," Circ. Res. 25(6), 637649 (1969).

${ }^{7}$ W. R. Milnor, "Arterial impedance as ventricular afterload," Circ. Res. 36(5), 565-570 (1975).

${ }^{8}$ A. P. Avolio, "Multi-branched model of the human arterial system," Med. Bio. Eng. Comput. 18, 709-718 (1980).

${ }^{9}$ J. E. Tsitlik, H. R. Halperin, A. S. Popel, A. A. Shoukas, F. C. P. Yin, and N. Westerhof, "Modeling the circulation with three-terminal electrical networks containing special non-linear capacitors," Ann. Biomed. Eng. 20, 595-616 (1992).

${ }^{10}$ D. N. Ku, "Blood flow in arteries," Annu. Rev. Fluid Mech. 29, 399-434 (1997).

${ }^{11}$ M. F. O'Rourke and J. Hashimoto, "Mechanical factors in arterial aging: A clinical perspective," J. Am. Coll. Cardiol. 50(1), 1-13 (2007).

${ }^{12}$ M. R. Mirzaee, O. Ghasemalizadeh, and B. Firoozabadi, "Exact simulating of human arteries using lumped model and probing constriction in femoral and carotid arteries," Am. J. Appl. Sci. 6(5), 834-842 (2009).

${ }^{13}$ M. Florens, B. Sapoval, and M. Filoche, "Optimal branching asymmetry of hydrodynamic pulsatile trees,” Phys. Rev. Lett. 106, 178104 (2011).

${ }^{14}$ C. Silva and A. Heitor Reis, "Scaling laws of branching pulsatile flows" J. Therm. Sci. (to appear).

${ }^{15}$ I. Voges, M. Jerosch-Herold, J. Hedderich, E. Pardun, C. Hart, D. D. Gabbert, H. H. Kramer, and C. Rickers, "Normal values of aortic dimensions, distensibility, and pulse wave velocity in children and young adults: A cross-sectional study," J. Cardiovasc. Magn. Reson. 14(Suppl. 1), O55 (2012).

${ }^{16}$ J. Sugawara, K. Hayashi, T. Yokoi, and H. Tanaka, "Age-associated elongation of the ascending aorta in adults," J. Am. Coll. Cardiol. Imaging 1, 739-748 (2008).

${ }^{17}$ A. R. Ahlgren, F. Hansen, B. Sonesson, and T. Lanne, "Stiffness and diameter of the common carotid artery and abdominal aorta in women," Ultrasound Med. Biol. 23, 983-988 (1997).

${ }^{18}$ A. Benetos, S. Laurent, A. P. Hoeks, P. H. Boutouyrie, and M. E. Safar, "Arterial alterations with aging and high blood pressure: A noninvasive study of carotid and femoral arteries," Arteriosclerosis, Thrombosis, and Vascular Biology 13, 90-97 (1993).

${ }^{19}$ B. Canaud, I. Jaussent, A. Rodriguez, H. Leray-Moragues, L. Chenine, A. Picard, M. Morena, and J. P. Cristol, "Whole-blood viscosity 
increases significantly in small arteries and capillaries in hemodiafiltration: Does acute hemorheological change trigger cardiovascular risk events in hemodialysis patient?," Hemodialysis Int. 14, 433-440 (2010).

${ }^{20}$ A. Bejan, Advanced Engineering Thermodynamics, 2nd ed. (Wiley, New York, 1997), Chap. 13.

${ }^{21}$ A. H. Reis, A. F. Miguel, and M. Aydin, "Constructal theory of flow architectures of the lungs," Med. Phys. 31(5), 1135-1140 (2004).

${ }^{22}$ A. Bejan and S. Lorente, "The constructal law and the evolution of design in nature," Phys. Life Rev. 8, 209-240 (2011).

${ }^{23}$ A. H. Reis and A. Bejan, "Constructal theory of global circulation and climate," Int. J. Heat Mass Transf. 49(11-12) 1857-1875 (2006).

${ }^{24}$ A. H. Reis, "Constructal view of scaling laws of river basins," Geomorphology 78, 201-206 (2006).

${ }^{25}$ A. H. Reis and C. Gama, "Sand size versus beachface slope - an explanation based on the Constructal Law," Geomorphology 114, 276-283 (2010).

${ }^{26}$ A. Bejan, Shape and Structure, From Engineering to Nature (Cambridge University Press, Cambridge, UK, 2000).

${ }^{27}$ A. H. Reis, "Constructal theory: From engineering to physics, and how flow systems develop shape and structure,” Appl. Mech. Rev. 59(5), 269282 (2006).

${ }^{28}$ A. Bejan and S. Lorente, Design with Constructal Theory (Wiley, Hoboken, 2008)
${ }^{29}$ A. Bejan and S. Lorente, "Constructal law of design and evolution: Physics, biology, technology, and society,” J. Appl. Phys. 113, 151301 (2013).

${ }^{30}$ C. Giannattasio, A. Vincenti, M. Failla, A. Capra, A. Ciro, S. De Ceglia, G. Gentile, R. Brambilla, and G. Mancia, "Effects of heart rate changes on arterial distensibility in humans," Hypertension 42, 253-256 (2003).

${ }^{31}$ A. Pitcher, P. Leeson, C. Forfar, C. Trevitt, J. M. Francis, S. Neubauer, and S. E. Petersen, "Aortic distensibility decreases during exercise in normal volunteers,” J. Cardiovasc. Magn. Reson. 12, P136 (2010).

${ }^{32}$ C. D. Murray, "The physiological principle of minimum work: I. The vascular system and the cost of blood volume," Proc. Natl. Acad. Sci. U.S.A. 12(3), 207-214 (1926).

${ }^{33}$ C. D. Murray, "The physiological principle of minimum work: II. Oxygen exchange in capillaries," Proc. Natl. Acad. Sci. U.S.A. 12(5), 299-304 (1926).

${ }^{34}$ T. F. Sherman, "On connecting large vessels to small: The meaning of Murray’s law,” J. Gen. Physiol. 78(4), 431-453 (1981).

${ }^{35}$ O. K. Baskurt and H. J. Meiselman, "Blood rheology and hemodynamics," Semin. Thromb. Hemostasis 29, 435-450 (2003).

${ }^{36}$ M. O'Rourke, A. Farnsworth, and J. O'Rourke, "Aortic dimensions and stiffness in normal adults," JACC: Cardiovasc. Imaging 1(6), 749-751 (2008). 